\title{
CHANGES IN RELATIVE GROWTH RATE WITH PLANT ONTOGENY IN SPRING WHEAT GENOTYPES GROWN AS ISOLATED PLANTS
}

\author{
C. J.T.SPITTERS ${ }^{1 *}$ and Th.KRAMER ${ }^{2}$ \\ 'Department of Theoretical Production Ecology, Agricultural University, Wageningen, the Netherlands \\ ${ }^{2}$ Institute of Plant Breeding (IvP), Agricultural University, Wageningen, the Netherlands
}

Received 4 May 1984

\begin{abstract}
INDEX WORDS
Triticum aestivum, wheat, growth analysis, relative growth rate, selection.

SUMMARY

For a better insight in the effect of wide spacing on the outcome of plant selection in spring wheat, the growth of free-grown individual plants was followed in time for each of 12 cultivars. As time proceeded, the cultivar differences for per-plant weight showed progressively less relation with those for seedling weight. At anthesis, the genetic correlation with seedling weight was still about 0.80 , but at final harvest it was only 0.06 . This contrasted with the genetic correlation between seedling weight and final biomass in closely planted mixtures which was on the average 0.77 . The outcome of selection of genotypes in isolation is therefore expected to deviate substantially from that of selection in densely planted segregating populations where the differences in initial size tend to be maintained in time.

The ranking of the varieties grown in isolation changed in time because of differences in relative growth rate (RGR). The genetic variance of RGR decreased much less with time than RGR itself. Late-flowering varieties showed the higher RGR so that these varieties improved their position in the ranking in isolation.

Special attention is paid to the methodology of plant growth analysis in variety experiments and in estimating means and variances of RGR.
\end{abstract}

\section{INTRODUCTION}

Competition between the different genotypes in a segregating population affects their yield and, with that, biases the outcome of selection for monoculture yield. It has often been advocated to use single-grown plants in selection in order to avoid the bias from intergenotypic competition between the plants (e.g. Fasoulas \& TSAFTARIS, 1975). However, in this way, the bias from intergenotypic competition is replaced by a bias from a differential response of the genotypes to the free space (SPITTERs, 1979, p. 76,98$)$. This prompts a study of the effect of spacing on the ranking of genotypes in mixture.

Experimental results indicated that relative differences in per-plant weight between genotypes of a summer annual tend to remain constant in time when they are grown in mixture with each other. This result was explained with a simple, causal model

* Present address: Foundation for Agricultural Plant Breeding, SVP, P.O. Box 117, NL-6700 AC Wageningen, the Netherlands. 
(SPITTERS, 1984; SCHAPENDONK \& SPITTERS, 1984). So the final biomass of a genotype, when competing with other genotypes, corresponds closely with its seedling weight. It may be expected, however, that the wider the spacing, the more apparent the genetic differences in coping with free space. These latter differences, probably related to ontogenic differences during the maturation phase, change the ranking of the genotypes when grown in isolation as compared with their ranking in competition. Thus, the degree of which the ranking of genotypes, when grown as isolated plants, is maintained in course of their growth will strongly determine the correspondence between different spacings in the outcome of selection.

To study the effect of wide spacing, growth of isolated plants of 12 spring wheat varieties was observed in time in the field. To describe the progression of plant weight in time, first a mathematical function has to be selected. This facilitates the prediction of plant weight and its derived quantities at any time of the growth period. The degree to which the differences among the genotypes change with time is quantified by the correlation between the seedling weights of the varieties and their plant weights at successive times. As changes in the ranking of the varieties are, by definition, brought about by differences in their relative growth rate (RGR), the time course of RGR and of the genetic variance in RGR is considered. Much attention is paid to the methodology of plant growth analysis because of the many pitfalls in this type of analysis, especially when applied to experiments with small differences between the genotypes and when the aim is to estimate population parameters as genetic variances and covariances.

\section{EXPERIMENTAL DESIGN}

To study the growth of isolated plants, widely spaced plants of 12 spring wheat varieties were harvested periodically. Commercial cultivars were involved as well as advanced breeding lines, all adapted to Dutch growing conditions. The seeds of the varieties were accurately spaced by hand in the field, two kernels being sown at each place and the plants being singled after emergence. The average day of emergence was 16 April 1980. The soil was a sandy clay loam. Weather data are given in the Appendix of SPITTERS \& KRAMER (1985).

For the harvest at days 16, 22, 28, 37 and 43 after emergence, plants were grown at a spacing of $15 \times 15 \mathrm{~cm}^{2}$. The harvests at days $22,28,37$ and 43 were laid out each as a randomized block design with 4 replicates and the 12 varieties randomized within the replicates. The net plot size consisted of 11 plants. The harvest at day 16 was a sample of, on the average, 86 representative plants of each of the varieties from the thinnings.

For the harvests at days $34,49,65$ and 80 , plants were grown at $20 \times 40 \mathrm{~cm}^{2}$. Each harvest was laid out as a randomized block design with 3 replicates (days 34 , 49,65 ) or 6 replicates (day 80 ). A net plot consisted of a row of 20 plants (day 34 ), 15 plants (days 49 and 65) or 5 plants (day 80 ) with $20 \mathrm{~cm}$ between the plants within the row and $40 \mathrm{~cm}$ between the rows.

For the final harvest at day 135 , plants were grown at $40 \times 40 \mathrm{~cm}^{2}$ in a randomized block design with 44 replicates of single plants.

Statistical analyses were based on plot means. The spacings were laid out adjacent 
Table 1. Dry weights and photosynthesizing areas of individual, isolated plants averaged over 12 varieties. The coefficient of variation $\mathrm{CV}_{\mathrm{e}}$ is that for total plant weight on a per-replicate base.

\begin{tabular}{|c|c|c|c|c|c|c|c|c|c|}
\hline \multirow{3}{*}{$\begin{array}{l}\text { Day } \\
\text { after } \\
\text { emer- } \\
\text { gence }\end{array}$} & \multicolumn{5}{|c|}{ Dry weight (g/plant) } & \multicolumn{2}{|c|}{ Green area $\left(\mathrm{cm}^{2} /\right.$ plant $)$} & \multirow{3}{*}{$\begin{array}{l}\mathrm{CV}_{\mathrm{e}} \\
\text { per } \\
\text { repli. }\end{array}$} & \multirow{3}{*}{$\begin{array}{l}\text { Number } \\
\text { of } \\
\text { repli- } \\
\text { cates }\end{array}$} \\
\hline & \multirow[t]{2}{*}{ total } & \multicolumn{2}{|c|}{ lear blades } & \multirow{2}{*}{$\begin{array}{l}\text { stems + } \\
\text { sheaths }\end{array}$} & \multirow[t]{2}{*}{ ears } & \multirow[t]{2}{*}{ leaves } & \multirow[t]{2}{*}{ ears ${ }^{1}$} & & \\
\hline & & green & yellow & & & & & & \\
\hline 16 & 0.0226 & . & & . & & . & & & 1 \\
\hline 22 & 0.0519 & 0.0362 & & 0.0157 & & 7.61 & & 0.041 & 4 \\
\hline 28 & 0.1246 & 0.0842 & & 0.0404 & & 18.30 & & 0.055 & 4 \\
\hline 34 & 0.580 & 0.355 & & 0.224 & & 77.9 & & 0.027 & 3 \\
\hline 37 & 0.705 & 0.413 & & 0.292 & & 88.2 & & 0.037 & 4 \\
\hline 43 & 1.281 & 0.685 & 0 & 0.596 & & 141.5 & & 0.057 & 4 \\
\hline 49 & 4.00 & 1.80 & 0.005 & 2.20 & 0 & 424 & 0 & 0.029 & 3 \\
\hline 65 & 14.48 & 3.34 & 0.02 & 8.88 & 2.25 & 749 & 110 & 0.034 & 3 \\
\hline 80 & 28.33 & 5.65 & 0.23 & 16.24 & 6.22 & 1018 & 208 & 0.034 & 6 \\
\hline 135 & 82.3 & 0 & . & . & $47.1^{2}$ & 0 & 0 & 0.031 & 11 \\
\hline
\end{tabular}

1 Two times the one-sided area measured.

${ }^{2}$ Of which $32.5 \mathrm{~g}$ grains.

to each other. The design is somewhat irregular because the experiment was part of a bigger experiment with several objectives.

\section{FITTING A FUNCTION TO THE GROWTH OF SINGLE GROWN PLANTS}

First a function is selected to describe the increase of weight in time for single-grown plants. Such a fitted function smoothes the variation which occurs in experimental data, simplifies the description of the progression of growth and facilitates the interpolation of plant weights of the varieties for any desired time.

Backgrounds and biometry of fitting curves to plant growth data were discussed by CAUSTON \& Venus (1981) and Hunt (1982). Some essential points appear also in the following discussion of the growth curves.

General methodology. In the process of curve fitting, the sum of the squared deviations of the actual data from the fitted values is minimized. Implicitly it is assumed that the variance of the data is the same for each harvest and that within a harvest the data follow a normal distribution. However the variance of the weight per plant increases strongly with increased plant size. In contrast to the variance, the coefficient of variation $(\mathrm{CV}=$ standard deviation/mean) remains about constant in course of time, so that the curves are fitted to $\ln$-transformed yield data in order to secure the assumption of homogeneity of variances.

Input were data per replicate rather than the means over replicates. Data per replicate have the maximum amount of information, as can be understood from the respective numbers of degrees of freedom. Moreover, in this experiment, CV on a per-replicate basis was more constant over time than $\mathrm{CV}$ of means across replicates (Table 1). For the final harvest the number of replicates was numerically reduced from 44 to 11 by grouping four plants per replicate in order to fulfil the requirement of a homo- 

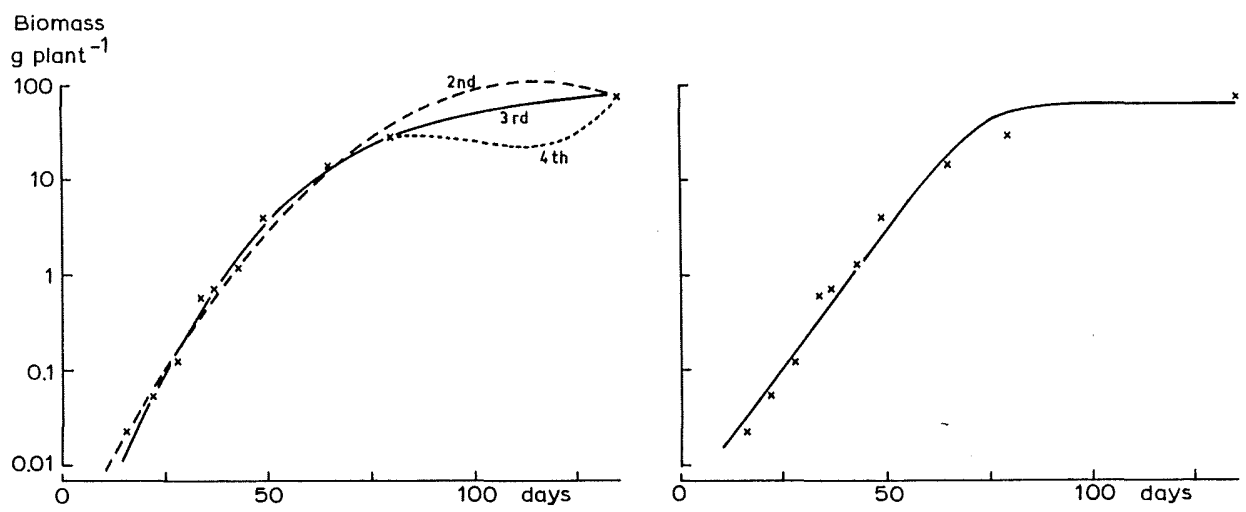

Fig. 1. Second, third and fourth degree polynomial functions (left) and Richards function (right) fitted through the In-transformed weights of plants grown in isolation. Input for the regression were the values per replicate (plot means) averaged over 12 varieties. The points in the graphs refer to means within dates of harvest.

geneous $\mathrm{CV}$. Note that because of their larger number of replicates the last two harvests had substantially greater weights when fitting the curve than other harvests.

Performance and characteristics of the two main groups of growth curves, the polynomials and the sigmoid curves, are discussed with fitting these curves to the plant weights averaged over all 12 varieties.

\section{Polynomial functions. The general form of the polynomial function is}

$\ln \mathrm{W}_{\mathrm{t}}=\mathrm{b}_{0}+\mathrm{b}_{1} \mathrm{t}+\mathrm{b}_{2} \mathrm{t}^{2}+\ldots .+\mathrm{b}_{\mathrm{n}} \mathrm{t}^{\mathrm{n}}$

where $W_{t}$ is the weight per plant at time $t$ and the b's are the regression coefficients. Because the natural logarithm of $\mathrm{W}$ is taken as a polynomial function of time, W itself is a polynomial exponential function of time. The coefficients are estimated by a multiple linear regression procedure.

The optimum polynomial degree is determined on the one hand by statistically objective criteria (CAUSTON \& VENUS, 1981, p. 85; HUnT, 1982, p. 112), and on the other hand by subjective considerations of the experimentalist. With respect to the statistical criteria, exte: ssion of the polynomial to a fourth degree yielded indeed a significant contribution of the fourth term $(P=0.006)$, but the reduction of the residual mean square was only $19 \%$ of that achieved by going from a second to a third degree. Fifth and higher powers did not give a significantly better fit. Subjective considerations in the choice of the polynomial degree are mathematical simplicity and biological sensibility. The lower the degree, the easier the function can be interpreted. For the studied data set, a second degree polynomial, i.e. a linear decrease of the relative growth rate with time, seemed an over-simplification. Moreover, the decrease of plant weight between day 115 and day 135 as predicted with this polynomial (Fig. 1) looks unrealistic. The fourth degree polynomical gave the better fit, but the interpolation between the harvests at days 80 and 135 (Fig. 1) is biologically unsatisfactory. As a result, progression of plant weight was described best by the third degree polynomial. For the plant weights, averaged over all 12 varieties, this polynomial is given by: 
$\ln \mathrm{W}_{\mathrm{t}}=-8.31+0.292 \mathrm{t}-0.00233 \mathrm{t}^{2}+0.0000064 \mathrm{t}^{3}$

The advantage of polynomial functions is that they are mathematically relatively simple and flexible. The coefficients are estimated with straightforward linear regression. However, with the exception of the first degree polynomial for exponential growth, they are purely empirical and do not have any biological basis.

Sigmoid functions. When one has no idea at all about the expected time trend, some polynomial function may be fitted to the data. However, many growth studies have proven that the weight per plant follows an S-shaped, asymptotic course in time, i.e. a sigmoid curve. The sigmoid curves have some physiological rationale and the parameters can be interpreted in biological terms. However, the mathematics is more difficult as the function is non-linear in the parameters.

RICHARDs (1959) presented a formula for a family of sigmoid curves. This Richards function is given by

$\mathrm{W}_{\mathrm{t}}^{1-\mathrm{m}}=\mathrm{A}^{1-\mathrm{m}}\left(1 \pm \mathrm{be}^{-\mathrm{kt}}\right)$

where $\mathrm{W}_{\mathrm{t}}$ is again weight per plant at time $\mathrm{t}$, e is the base of the natural logarithm, and $\mathrm{A}, \mathrm{b}, \mathrm{k}$ and $\mathrm{m}$ are constants to be estimated. The plus sign applies when $\mathrm{m}>$ 1. The minus sign when $0 \leqslant \mathrm{~m}<1$, while the function is not defined for $\mathrm{m}<0$, nor for $\mathrm{m}=1$. Substitution of $\mathrm{m}=2$ gives the logistic function, $\mathrm{m}=0$ the monomolecular function, and when $m$ approaches unity the function approaches to that of Gompertz (RICHARDS, 1959, 1969).

The Richards function was fitted to the ln-transformed data with a least-squares optimalisation technique for non-linear functions. For the progression of plant weight averaged over all 12 varieties a curve was found with $\mathrm{m}=2.24, \mathrm{~b}=161378, \mathrm{k}=$ 0.164 day $^{-1}$, and $\mathrm{A}=63.3 \mathrm{~g} \mathrm{plant}^{-1}$ (Fig. 1). As $\mathrm{m}$ is close to 2, this function differed very little from a simple logistic function. The interpretation of the regression coefficients and the mathematics of this function is found in RICHARDS $(1959,1969)$ and Causton \& Venus (1981, Chapter 4). By substituting $\mathrm{m}=\mathrm{v}+1, \mathrm{k}=\mathrm{\kappa}$ and $\mathrm{b}=$ $\exp (\beta)$, the notation used by the latter authors is obtained.

Compared with the polynomials, the Richards function gave the lesser fit of the experimental data (Fig. 1). For that part of the variation among the input data which is accounted for by the fitted function $\left(\mathrm{R}^{2}\right)$, we found 0.996 for the third and fourth degree polynomials, 0.992 for the second degree polynomial and only 0.982 for the Richards function.

The inferior fit of the latter is due to its poor description of the general form of the growth curve. The observed time trend was that of a long phase where growth was about exponential (linear increase at ln-scale), which was maintained because of the improving growth conditions of increasing temperature and daily radiation. After this phase with a near constant relative growth rate, the relative growth rate slowed down gradually (as characterized by the slopes of the curves in Fig. 1). However, for $\mathrm{m}>1$ the Richards function assumes an S-shaped decrease of the relative growth rate in time, which decrease is symmetrical around its point of inflection midway between the two asymptotes $\mathrm{k} /(\mathrm{m}-1)$ and 0 (CAuston \& Venus, 1981, p. 97-98). The observed trend clearly did not show this symmetry. 
Partly because of the observed asymmetry, the lack of fit was large for the final harvest, although the number of replicates of this harvest was by far the highest. For the final harvest a weight of $63 \mathrm{~g} \mathrm{plant}^{-1}$ was predicted whereas the observed geometric average was $80 \mathrm{~g} \mathrm{plant}^{-1}$ with a range of 69 to $98 \mathrm{~g} \mathrm{plant}^{-1}$ among the 12 varieties. The third degree polynomial predicted a final weight of $81 \mathrm{~g} \mathrm{plant}^{-1}$.

The experiment dealt with single-grown plants. At normal, closer spacings ripening is speeded up and then the time trend for the relative growth rate may approach more closely the symmetry underlying the Richards functions.

\section{COMPARING THE GROWTH OF VARIETIES IN COURSE OF TIME}

Comparison based on functions fitted to the plant weights of each variety separately. Given the performance of the functions, a third degree polynomial seemed most suitable to describe the growth of each of the varieties in this experiment. Comparison of the varieties will, therefore, be based to a great extent on third degree polynomials fitted to the ln-transformed plant weights of each variety separately.

However, some comments have to be made regarding the use of these fitted functions:

(a) The error around a fitted function is greatest at both its ends. These ends, the initial and the final weight, are, however, the most important parts with respect to our purpose.

(b) Differences between predicted and observed values do not only consist of random variation in the observations, but they consist also of a systematic lack of fit. These systematic deviations may, for example, arise from improper description of the general shape of the time trend, as appeared to occur for the Richards function, and by an over-estimation of growth in dull periods. The errors in each of the fitted functions accumulate partly in comparing the fitted functions with each other, e.g. in ratios or differences of plant weights between varieties. This may become troublesome, especially for interpolations between harvests.

Thus, although the comparison of varieties from functions fitted to the plant weights of each of them has several advantages, especially the ease in which interpolations can be made, one should exercise caution.

Comparison based on the deviation of each variety from the mean across all 12 varieties. Mutual comparison of the varieties based on the fitted polynomials of each, was not very clarifying. Plotting their differences against time was more illustrative.

One may use either differences of observed plant weights or differences of plant weights predicted from fitted functions. Use of observed differences avoids the accumulation of the systematic errors confounded with fitted functions, but includes random variation so that interpolation between harvests becomes more difficult. In this section the observed differences were used, partly in order to illustrate this approach and partly because a difference of two fitted functions appeared to be less satisfactory than a function fitted to the observed difference.

Because interest is in the change of the relative differences between the varieties over time, we take for each replicate the difference of the ln-transformed data 

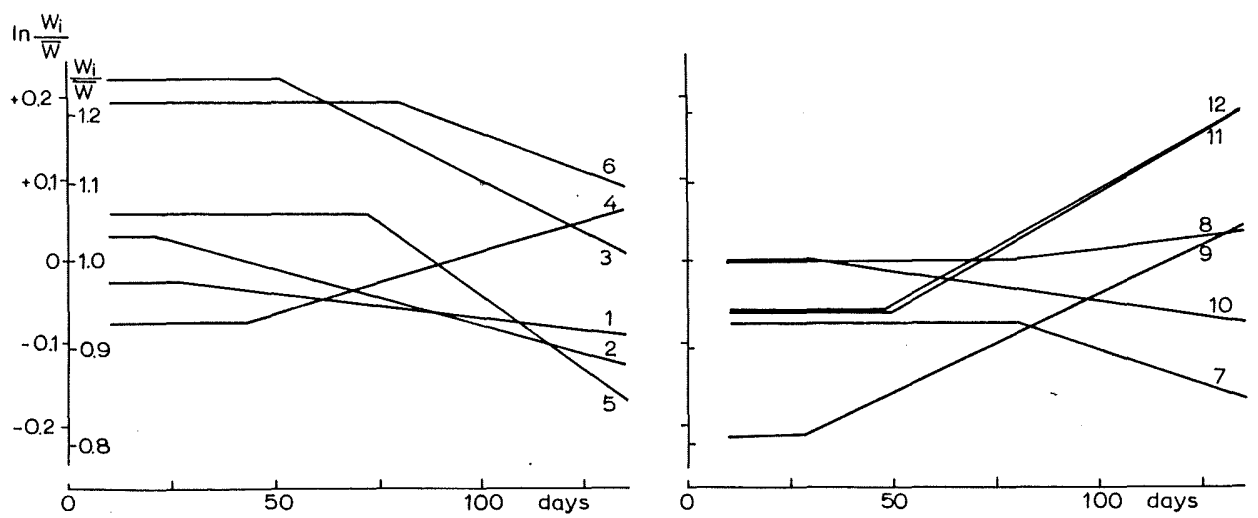

Fig. 2. Progress in time of the per-plant weights $\mathrm{W}_{\mathrm{i}}$ of each of 12 varieties related to the average weight $\overline{\mathrm{W}}$ of all varieties at that time. Time trends are fitted by Blackman curves. The numbers refer to the rank of the varieties used in Tables of Spitters \& Kramer (1985).

$\Delta \ln \mathrm{W}_{\mathrm{i}, \mathrm{t}}=\ln \mathrm{W}_{\mathrm{i}, \mathrm{t}}-\overline{\ln \mathrm{W}_{\mathrm{t}}}=\ln \left(\mathrm{W}_{\mathrm{i}, \mathrm{t}} / \overline{\mathrm{W}_{\mathrm{t}}}\right)$

where $\mathrm{W}_{\mathrm{i}, \mathrm{t}}$ is per-plant weight of variety $\mathrm{i}$ at time $t$ and $\overline{\mathrm{W}}$ the weight averaged geometrically over all varieties. To smooth the scatter in the data points, a time trend was fitted to these data.

Use of polynomial functions to fit the time trends of $\Delta \ln \mathrm{W}_{\mathrm{i}, \mathrm{t}}$ resulted in curves which were difficult to interpret. Over-fitting, i.e. unrealistic interpolation between harvests was frequently observed. Therefore some assumptions about the trend of $\Delta \ln \mathrm{W}_{\mathrm{i}, \mathrm{t}}$ were made in order to arrive at a more understandable function.

It was supposed that differences between the varieties in relative growth rate can be neglected for the initial growth stages, but that after some time such differences arise (a) because of a different pattern of dry matter allocation within the plant, especially as a result of genetic diversity in rate of development, and (b) because of different rates of senescence. This implies that relative biomass differences between the varieties remain constant for a short time, but that thereafter they change gradually. Under the simplifying assumption that the direction of the change remains constant, either improving or worsening the position of a variety, asymptotic types of curves are obtained for the time trends of $\Delta \ln \mathrm{W}_{\mathrm{i}, \mathrm{t}}$ (Fig. 2).

Three asymptotic type functions were fitted for each variety separately with a leastsquares optimalisation technique for non-linear functions. Input were the $\Delta \ln \mathrm{W}_{\mathrm{i}, \mathrm{t}}$ values of individual replicates.

The Blackman function as used in Fig 2. gave the best fit $\left(\mathrm{R}^{2}=0.797\right)$, followed by the asymptotic exponential $\left(\mathrm{R}^{2}=0.787\right)$ and at the final place the rectangular hyperbola $\left(\mathrm{R}^{2}=0.784\right)$. (In the calculation of $\mathrm{R}^{2}$, the sum of squares between replicates was removed from the residual sum of squares around the fitted function.) This ranking of the three types of functions was remarkably consistent over the 12 varieties, suggesting a common shape of the curves. This ranking coincides with decreasing sharpness of the curvature with the Blackman function showing the sharpest curve. Besides its superior fit, the Blackman function has the advantage of being easier to 
interpret and less difficult to fit. After dividing the time trend of $\Delta \ln \mathrm{W}_{\mathrm{i}, \mathrm{t}}$ by hand into a horizontal and an inclining section, the $\Delta \ln \mathrm{W}_{\mathrm{i}, \mathrm{t}}$ data might be fitted also to simple linear regression lines. Linear regression of the data of the entire growth period may even be a more simple alternative.

With saturation type functions, modifications in the position of a variety are represented only in one direction. Temporary changes towards the other direction, which are expected to occur especially during early growth, are obscured. For example, in this experiment, the genetic differences in relative growth rate, the factor responsible for changes in rank, over the period of days 22 to 37 had little relation with those in the later intervals of days $49-65,65-80$ and $80-135\left(\mathrm{r}_{\mathrm{g}}{ }^{*}=0.05\right.$ on the average). On the other hand, the relative growth rates in these latter intervals corresponded quite closely with each other $\left(\mathrm{r}_{\mathrm{g}}=0.61\right.$ on the average; Table 3 of SPITTERs \& KRAMER, 1985).

The method proposed gives, altogether, a first description of the changes in the relative plant weight differences between the varieties in time.

\section{CORRELATION OF PLANT WEIGHT WITH SEEDLING WEIGHT}

The approach of Fig. 2 is especially useful when one is interested in the performance of individual genotypes, e.g. when the aim is to select some variety. For general conclusions, however, one should look for a quantity characterizing the overall trend in the population. The coefficient of the correlation between the seedling weight of a variety and its weight at later times is such a quantity. This coefficient characterizes the degree to which the varietal differences are maintained in the course of the ontogeny.

Estimation method. The inital status of the varieties, their starting weight, was estimated by their ln-transformed plant weights averaged over the harvests at days 16,22 and 28. So the starting weights represent seedling weights at the average day 22 . These starting weights were correlated with per-plant weights at each harvest. The natural logarithms of the weights were used because ratios, rather than absolute differences were the aim of the study (Equation 1). For the correlations involving one of the first three harvests, the contribution of the harvest itself to the starting weight was removed in order to avoid an inflation of the correlation due to an autocorrelation.

A correlation between variety means is deflated by the random variation inherent to each variety mean. With that, it is a phenotypic correlation affected by factors like sample size. From the definition of the correlation coefficient and the partitioning of phenotypic value into contributions because of genotype and environment $(\mathrm{p}=$ $\mathrm{g}+\mathrm{e})$, the phenotypic correlation coefficient can be extended to

$r_{p}=\frac{\operatorname{cov}\left(g_{x}, g_{y}\right)+\operatorname{cov}\left(e_{x}, e_{y}\right)}{\sqrt{\operatorname{var} p_{x} \cdot \operatorname{var}} p_{y}}$

with $\mathrm{x}$ and $\mathrm{y}$ the correlated characteristics and assuming absence of a correlation be-

* In this paper, correlation coefficients based on variety means have the subscript $\mathrm{p}$ of phenotype. Because of the random variation in the means, these correlations are lower than the real genetic correlations, represented by $r_{\mathrm{g}}$ and defined in Equation 3 . 


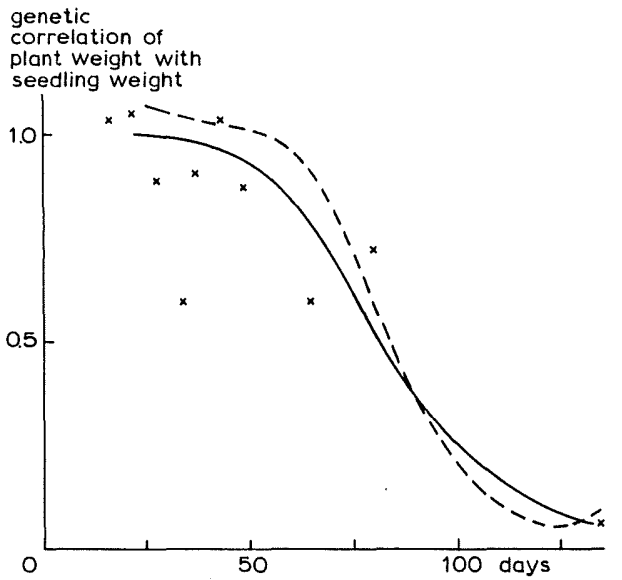

Fig. 3. Decrease in time of the genotypic correlation of the weight of single-grown plants with their seedling weights at day 22 . The broken curve is based on plant weights predicted from a third degree polynomial fitted to the observed plant weights. The solid curve was drawn by hand (see text).

tween genotype and environment. The variance among variety means (var $\mathrm{p}$ ) equals the sum of the genetic variance (var g) and the environmental variance (var e). From Equation 2, the 'genetic' correlation coefficient can be derived:

$r_{g}=\frac{r_{p} \sqrt{\operatorname{var} p_{x} \cdot \operatorname{var} p_{y}}-r_{e} \sqrt{\operatorname{var}_{x} \cdot \operatorname{vare}_{y}}}{\sqrt{\operatorname{var}_{x} \cdot \operatorname{var}_{y}}}$

When the error terms are mutually independent, which will in general be the case for different harvest, $r_{e}=0$. The variances were estimated from an analysis of variance. Note that var e refers here to the random variation around a mean and is therefore obtained by dividing the error variance found with the analysis of variance by the number of replicates on which that mean is based.

Results and discussion. The genetic correlation of plant weight with seedling weight is plotted for different dates of harvest in Fig. 3. Owing to random variation, estimates greater than unity may follow from Equation 3. On the average, $r_{g}$ was 1.3 times $r_{p}$.

Using plant weights predicted with a fitted function, rather than the observed weights, admits of obtaining estimates of $r_{g}$ at any desired time. For that purpose the data set was divided into three replicates. For each variety in each replicate, a third degree polynomial was fitted to the 14 values for $\ln W$. With time steps of 5 days, $\ln \mathrm{W}$ values predicted with the polynomials were submitted to an analysis of variance and were correlated with the actual seedling weight. Then the genetic correlation was derived according to Equation 3. The resulting trend of $r_{g}$ is given in Fig. 3 by the broken curve.

Some comments have to be made on the estimated correlations. (a) The confidence interval of the single data points in Figure 3 is very wide. Moreover, the values for $r_{g}$ tend to be slightly underestimated because the environmental variance used was that within dates of harvest. As the different harvests were not randomized within blocks but occurred as main blocks grown adjacent to each other, the variance between the harvest blocks should be added to the environmental variance and in that way removed from $r_{\mathrm{g}}$. (b) The error terms of the values predicted from the fitted function (broken curve in Figure 3) are mutually dependent because any point of the fitted 
growth function contains information of all harvests. This introduces a positive $r_{e}$ in Equation 3. This correlation becomes greater as time approaches day 22, the average harvest date on which the seedling weight is based. As no account was taken for this 'environmental' correlation, the genetic correlation coefficients tend to be somewhat overestimated, the more time approaches day 22. There were no harvests between days 80 and 135. The broken curve of Fig. 3 suggests that some over-fitting occurred in the interpolation between these harvests.

The most definite values of $r_{g}$ are a value of one for day 22, the time for which the seedling weight was defined, and the observed value at final harvest as the data for that harvest showed the smallest coefficient of variation. Based on the above-mentioned considerations, the hand-fitted solid curve of Fig. 3 for $r_{g}$ was drawn.

Fig. 3 shows that the genetic correlation with initial weight decreased in time according to a mirror-image S-curve. During the vegetative growth, this correlation kept a high value of roughly 0.80 at anthesis at day 67 . After anthesis, the correlation declined rapidly. At final harvest, the ranking of the varieties showed no relation with that at seedling stage $\left(r_{\mathrm{g}}=0.06\right)$.

Relationship between the correlation coefficient and the relative growth rate. The relative growth rate is defined by

$\mathrm{RGR}=\frac{\mathrm{dW}_{\mathrm{t}} / \mathrm{dt}}{\mathrm{W}_{\mathrm{t}}}=\frac{\mathrm{dln}_{\mathrm{t}}}{\mathrm{dt}}$

The change in the deviation of plant weight $\mathrm{W}_{\mathrm{i}, \mathrm{t}}$ of variety i from the population mean $\overline{\mathrm{W}}_{\mathrm{t}}$, is then defined by

$\frac{\mathrm{d}\left(\ln \mathrm{W}_{\mathrm{i}, \mathrm{t}}-\ln \overline{\mathrm{W}}_{\mathrm{t}}\right)}{\mathrm{dt}}=\mathrm{RGR}_{\mathrm{i}}-\mathrm{RGR}_{\text {mean }}$

where $R G R_{\text {mean }}$ is the relative growth rate of plant weight averaged over varieties. Hence, varietal differences in instantaneous RGR are the direct cause of changes in the ranking of the varieties for the weight per plant. Thus, varietal differences in RGR fully explain time trens as depicted in Fig. 2.

The correlation between the per-plant weights $\mathrm{W}_{\mathrm{t}}$ of the varieties and their initial weights $\mathrm{W}_{0}$ is a function of the varietal differences in initial weight and in relative growth rate. This can be seen by substituting the equation for exponential growth

$\mathrm{W}_{\mathrm{t}}=\mathrm{W}_{0} \mathrm{e}^{\overline{\mathrm{RGR}} \cdot \mathrm{t}}$ or $\ln \mathrm{W}_{\mathrm{t}}=\ln \mathrm{W}_{0}+\overline{\mathrm{RGR}} \cdot \mathrm{t}$

into the definition of the correlation coefficient. This gives

$\mathrm{r}_{\ln _{0}, \ln \mathrm{W}_{\mathrm{t}}}=\frac{\operatorname{cov}\left(\ln \mathrm{W}_{0}, \ln \mathrm{W}_{\mathrm{t}}\right)}{\sqrt{\operatorname{var} \ln \mathrm{W}_{0} \cdot \operatorname{var} \ln \mathrm{W}_{\mathrm{t}}}}=\sqrt{\frac{\operatorname{var} \ln \mathrm{W}_{0}}{\operatorname{var} \ln \mathrm{W}_{0}+\mathrm{t}^{2} \operatorname{var} \overline{R G R}}}$

where in the latter step cov ( $\left.\ln \mathrm{W}_{0}, \overline{\mathrm{RGR}}\right)$ was supposed to be zero. $\overline{\mathrm{RGR}}$ denotes the mean relative growth rate over the entire period from emergence to time t. As the genetic correlation is considered, the genetic co-variance and the genetic variances are involved. From Equation 6, we see that the correlation with initial weight declines as time proceeds. The smaller the differences in initial weight and the greater the differences in $\overline{\mathrm{RGR}}$ the faster the decline. 


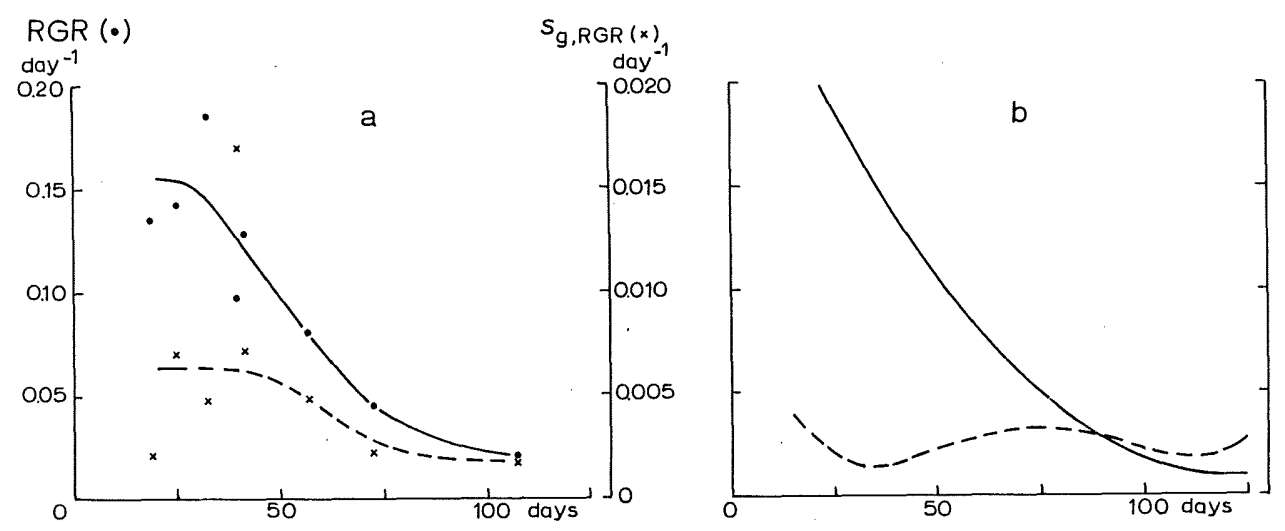

Fig. 4. Relative growth rate, its mean and genetic standard deviation over 12 varieties (a) Estimates for the successive harvest intervals and (b) estimates from the third degree polynomial functions fitted to the ln-transformed plant weights for each variety. Curves of (a) were hand fitted.

\section{RELATIVE GROWTH RATE, ITS MEAN AND GENETIC VARIATION}

As shown in the preceding section, varietal differences in relative growth rate are responsible for the changes in the ranking of the varieties in plant weight.

The mean relative growth rate over the time interval $t_{1}-t_{2}$ is calculated as

$\overline{\mathrm{RGR}}=\left(\ln \mathrm{W}_{2}-\ln \mathrm{W}_{1}\right) /\left(\mathrm{t}_{2}-\mathrm{t}_{1}\right)$

This method has already been used for several decades and is therefore called the 'classical approach'. The relative growth rate can also be calculated as the first derivative from a function fitted to the observed $\operatorname{lnW}$ values to time $t$ (Equation 4). This is called the 'functional approach'. This latter method gives instantaneous values of RGR.

Both approaches will be discussed. The results are presented in Fig. 4.

Classical approach. In the classical approach of Fig. 4a, $\overline{R G R}$ was estimated for each interval between two subsequent dates of harvest according to Equation 7. Estimations were done for each variety and each replicate separately. The resulting RGR's were submitted to an analysis of variance. This gave an estimate of $\overline{R G R}$ averaged over the 12 varieties and an estimate of the genetic variance around this average for each interval.

Tge discrimination of replicates implies pairing of $\ln W$ values of harvest $t_{1}$ with $\ln W$ values of harvest $t_{2}$. Pairing makes only sense when there are significant replicate effects, as was the case in this experiment. Otherwise, the best estimates of the variances of $\overline{R G R}$ are obtained directly from Equation 7 as:

$\operatorname{var} \mathrm{e}_{\overline{R G R}}=\left(\operatorname{var} \mathrm{e}_{\operatorname{lnW}}+\operatorname{var}_{\mathrm{lnW}_{2}}\right) /\left(\mathrm{t}_{2}-\mathrm{t}_{1}\right)^{2}$

and

$\operatorname{var} g_{\overline{R G R}}=\left(\operatorname{var} g_{\ln W_{1}}+\operatorname{var} g_{\ln W_{2}}-2 r_{p} \sqrt{\operatorname{var} p_{\ln W_{1}} \cdot \operatorname{var} p_{l n w_{2}}} / /\left(t_{2}-t_{1}\right)^{2}\right.$ 
where var $p$ refers to the variance among variety means of $\ln W$ and $r_{p}$ is the correlation between variety means of $\ln W$ at $t_{1}$ and $t_{2}$, respectively. The error terms at $t_{1}$ are supposed to be independent of those at $t_{2}$.

In this experiment the pairing and the non-pairing method yielded similar values.

Functional approach. The time trend of RGR in Fig. $4 \mathrm{~b}$ is the first derivative of the third degree polynomial represented in Fig. 1. To estimate the genetic variance of $\mathrm{RGR}$, a third degree polynomial was fitted to the $\ln \mathrm{W}$ values for each variety in each of the three installed replicates. The time trends of RGR are given by the first derivative of each of these functions. For each time step of 5 days, the 3 RGR values for each of the 12 varieties were submitted to an analysis of variance. From this an estimate of the genetic variance is obtained for each time step.

It is a wrong procedure to fit a function per variety over replicates and subsequently estimating the genetic variance as the difference between the variance among the variety means of RGR and the mean residual variance of RGR derived from the residual variances of the fitted growth functions. As explained before, this residual variance of RGR contains not only the random error variance but also a variance due to a bias in the model, i.e. the systematic deviations from the regression. In this way, var $e_{R G R}$ would be strongly overestimated so that highly deflated estimates of var $g_{R G R}$ result.

Comparison of the classical and the functional approach. The classical approach (Fig. 4a) has some disadvantages. (a) Each estimate, for the mean as well as for the standard deviation, is subject to a large random variation. The coefficient of variation of $\mathrm{s}_{\mathrm{g}, \mathrm{RGR}}$ was e.g. on the average $133 \%$ for the first four intervals. It decreased to $29 \%$ for the final harvest interval. (These approximate CV's were obtained by the method given by COMSTOCK \& MOLL (1963) to estimate the variance of an estimate of a between-class variance. More exact estimates of confidence intervals are obtained with the procedure presented by SNEDECOR \& COCHRAN (1967, p. 284-285).) The error around the, handfitted, time trend is smaller than that of individual values as such a trend may be interpreted to consist of incomplete replicates in time. (b) When, owing to random effects, the per-plant weight at $t_{2}$ is smaller than expected, the RGR over $t_{1}-t_{2}$ is underestimated whereas the RGR over $t_{2}-t_{3}$ is overestimated, and vice versa. In this way,a tendency to a negative correlation between RGR's of successive intervals is generated. In this experiment, an overestimation of the yield level at day 37 might be the cause of the deviating values of the intervals $28-37$ and 37-43.

With the functional approach (Fig. 4b), this negative correlation between RGR's of successive harvests is avoided. Furthermore, the confidence interval around the derived curves is narrower because information from all harvests is included in the fitted function. In the period between days 25 and 125 , the $\mathrm{CV}$ of $\mathrm{s}_{\mathrm{g}, \mathrm{RGR}}$ was about $33 \%$.

Comparison of Fig. $4 \mathrm{~b}$ with the data points in Fig. 4a suggests, however, that the functional approach generated some deviating results, at least for the early growth. For the early phases, RGR tended to be overestimated. This lack of fit of the third degree polynomial is now easier detected than in graphs like Fig. 1 where $\ln W$ is plotted 
against time. The genetic variation of RGR is probably underestimated for the early phase of growth by the functional approach, but a definite conclusion cannot be given because of the very wide confidence intervals of this quantity.

The time trend of RGR and of its genetic variation. RGR decreased in time (Fig. 4), a trend which is discussed in more detail in SPITTERS \& KRAMER (1985). The genetic variation for RGR is represented in Fig. 4 by $s_{g, R G R}$, the square root of the genetic variance of RGR. This presentation facilitates the comparison with RGR itself as both quantities have the same dimension. Contrary to RGR, $\mathrm{s}_{\mathrm{g}, \mathrm{RGR}}$ did not show an unanimous trend. The results derived from the fitted polynomial (Fig. 4b) suggest that $\mathrm{s}_{\mathrm{g}, \mathrm{RGR}}$ remained about constant in time.

The curves of RGR and $s_{g, R G R}$ converged. Hence, when time proceeded the varietal differences in RGR increased when they were expressed relative to the mean level of RGR. The genetic coefficient of variation $\left(\mathrm{CV}_{\mathrm{g}}\right)$ of RGR increased in time.

Order of magnitude of the genetic variation in RGR. In Fig. $4, \mathrm{~s}_{\mathrm{g}, \mathrm{RGR}}$ is plotted on a scale which is 10 times finer than that for RGR itself. This illustrates that the genetic differences in RGR were small compared to the level of RGR. The $\mathrm{CV}_{\mathrm{g}, \mathrm{RGR}}$ was in the order of $2-4 \%$ in the early growth stages and increased to $10 \%$ in the ripening stage. The median value for the observed $\mathrm{CV}_{\mathrm{g}, \mathrm{RGR}}$ was $5 \%$, when considered over the entire growth period (Fig. 4a).

The results give an impression of the sample size required to screen varieties for differences in RGR. According to Equation 7a, the random variance of RGR amounts to:

$\operatorname{var} \mathrm{e}_{\overline{R G R}}=\frac{2 \operatorname{var} \mathrm{e}_{\ln W}}{\left(\mathrm{t}_{2}-\mathrm{t}_{1}\right)^{2}}$

For the period between days 22 and 43 , var $\mathrm{e}_{\mathrm{ln} w}$ was on the average 0.25 when extrapolated from means per plot to a per-plant base. An expression for var $\bar{e}_{\overline{R G R}}$ for samples of $n$ plants per harvest can be derived under the assumptions that (a) the plants within a harvest are uncorrelated, which seems a reasonable assumption for plants grown without interplant competition, and (b) the varieties are sufficiently randomized to avoid a correlation with place (Spitters, 1979, p. 196-200). This gives for samples of $\mathrm{n}$ plants var $\mathrm{e}_{\overline{\mathrm{RGR}}}=2 \times 0.25 /\left(\mathrm{n}\left(\mathrm{t}_{2}-\mathrm{t}_{1}\right)^{2}\right)$. For $\overline{\mathrm{RGR}}=0.15 \mathrm{day}^{-1}$, this results in $\mathrm{CV}_{\mathrm{e}, \mathrm{RGR}}=4.7 /\left(\left(\mathrm{t}_{2}-\mathrm{t}_{1}\right) \sqrt{\mathrm{n}}\right)$. The size for the varietal differences in RGR to be expected is about $4 \%$, as derived from Fig. 4a. This value of $\mathrm{CV}_{\mathrm{g}, \overline{\mathrm{RGR}}}$ refers to the early growth of spring wheat varieties grown under field conditions. The required sample size and number of replicates can now be estimated with standard procedures (e.g. Cochran \& Cox, 1957, p. 17-23).

Comparison of mean against instantaneous values of $R G R$. The $\overline{R G R}$ for a certain time interval equals the mean of all instantaneous RGR values of that period. This implies for the variance of $\overline{R G R}$ that

$\operatorname{var} \overline{\mathrm{RGR}}=\frac{1}{\mathrm{~T}} \overline{\operatorname{var} R G R}+\frac{\mathrm{T}-1}{\mathrm{~T}} \overline{\operatorname{cov}\left(\mathrm{RGR}_{\mathrm{t}}, \mathrm{RGR}_{\mathrm{ij}}\right)}$ 
where $\mathrm{T}$ denotes the number of time steps (subintervals) in which the interval is subdivided, and $t_{i}$ and $t_{j}$ refer to two different times. Thus, the smaller the time steps, i.e. the larger $T$, the smaller the effect of var RGR on var $\overline{R G R}$ and the more important the co-variances between the RGR's of the different subintervals. On the other hand, with shorter time steps, there are relatively more related RGR's within the data set, viz. the RGR's of subsequent steps, which forces the average co-variance more towards the variance.

Equation 8 shows that the genetic variance based on instantaneous values of RGR (Fig. 4b) is not strictly comparable with that based on RGR's averaged over certain time periods (Fig. 4a). The difference is probably not large. Var $\bar{g}_{\overline{R G R}}$ was predicted from the third degree polynomial with $R$ GR the mean relative growth rate over the period from day 22, the second harvest, to day $t$ with $t$ running from days 22 to 135 . The resulting time trend of $\mathrm{s}_{\mathrm{g}, \mathrm{RGR}}$ differed little from that of $\mathrm{s}_{\mathrm{g}, \mathrm{RGR}}$ given in Fig. $4 \mathrm{~b}$ : the median value of $\mathrm{s}_{\mathrm{g}, \overline{R G R}}$ was 0.0019 against a median of 0.0023 for $\mathrm{s}_{\mathrm{g}, \mathrm{RGR}}$, but the fluctuations in time were strongly damped with the mean $\overline{R G R}$.

\section{DISCUSSION}

On theoretical and empirical grounds, it was expected that in a close-spaced mixture of different genotypes of a summer annual, the relative differences among genotypes in per-plant weight remain roughly the same during the growing season (SPITTERS, 1984). This was supported by the results of the present experiment where the genetic correlation between seedling weight and final plant weight was $0.84,0.81,0.70$ and 0.72 for mixtures grown at $5 \times 5,10 \times 10,15 \times 15$, and $20 \times 20 \mathrm{~cm}^{2}$ plant ${ }^{-1}$, respectively. These close-spaced mixtures will be discussed in a forthcoming paper.

On the other hand, success of individual, free-growing plants is not only determined by their initial status but also by their ability to cope with the free space. This resulted in a mirror-image S-shaped decline in time of the genetic correlation of plant weight with seedling weight. At anthesis, there was still good correspondence between the biomass of a variety and its seedling weight $\left(\mathrm{r}_{\mathrm{g}} \approx 0.80\right)$, but at final harvest there was no relation at all $\left(r_{g}=0.06\right)$ (Fig. 3 ).

The change in course of time of the ranking in plant weight of the varieties is caused, by definition, by differences in relative growth rate (Equation 6). Late-flowering varieties improved their position, measured by their share in the biomass total of the population, because they had the higher RGR. The correlation between the $\overline{R G R}$ of a variety and its date of anthesis was: $r_{g}=0.51,0.89$ and 0.72 with $\overline{R G R}$ referring to intervals 49-65, 65-80 and 80-135, respectively, with the average date of anthesis at day 67 after emergence. The substantial effect on $\overline{R G R}$ of relatively small differences in time of anthesis, only 7 days between the earliest and latest variety, suggests that for isolated plants anthesis is linked closely to the rate of development over an extended part of the ontogeny. The advantage of a later date of anthesis was presumably a later ripening, resulting in a greater assimilation capacity and therefore higher RGR during the maturation phase.

In the close-spaced mixtures, which were referred to, the different varieties ripened quite synchronously, probably forced by a depletion of a limited stock of water and 
nutrients (SPIERTZ \& VAN KEULEN, 1980) and the suppression of late ears due to competition for light. Due to the synchronization of ripening imposed by the environmental stress, the relative positions of the genotypes in the close-spaced mixtures changed little in time. This was shown by the high genetic correlation with seedling weight found in these mixtures.

On the other hand, at the very wide spacing, genetic differences in date of maturity could exhibit themselves fully because of the rich supply of the isolated plants with growth resources. So the ability to cope with free space seems to a large extent to be based on a late ripening. Selection in isolation benefits late-flowering and lateripening genotypes. The relation between the yields of the genotypes in isolation with their yields in mixture and monoculture at normal spacings was discussed by KRAMER (1984).

\section{REFERENCES}

Causton, D. R. \& J. C. Venus, 1981. The biometry of plant growth. Edward Arnold, London, $307 \mathrm{pp}$.

Cochran, W. G. \& G. M. Cox, 1957. Experimental designs. John Wiley \& Sons, New York, 2nd ed., $611 \mathrm{pp}$.

Comstock, R. E. \& R. H. Moll, 1963. Genotype-environment interactions. In: W. D. HaNSON \& H. F. Robinson (Eds), Statistical genetics and plant breeding. Nat. Acad. Sci., Nat. Res. Council, Washington DC, Publ. 982: 164-194.

Fasoulas, A. \& A. TSaftaris, 1975. An integrated approach to plant breeding and field experimentation. Dept Genet. Plant Breed., Aristotelian Univ., Thessaloniki, Greece, Publ. 5, 37 pp.

HunT, R., 1982. Plant growth curves. Edward Arnold, London, 248 pp.

Kramer, Th., 1984. Fundamental considerations on the density-dependence of the selection response to plant selection in wheat. Proc. 6th Int. Wheat Genetics Symp. Kyoto, Japan: 719-724.

Richards, F. J., 1959. A flexible growth function for empirical use. J. exp. Bot. 10: 290-300.

Richards, F. J., 1969. The quantitative analysis of growth. In: F. C. STEWARd (Ed.), Plant physiology. V A: Analysis of growth. Behavior of plants and their organs. Academic Press, New York, pp. 3-76.

SNedecor, G. W. \& W. G. Cochran, 1967. Statistical methods. Iowa State Univ, Press, Ames, Iowa, 6th ed., 593 pp.

SPIERTZ, J. H. J. \& H. VAN KeUlEN, 1980. Effects of nitrogen and water supply on growth and grain yield of wheat. Proc. 3rd Int. Wheat Conference, Madrid, pp. 595-610.

SCHAPENDONK, A. H. C. M. \& C. J. T. SPITTERS, 1984. Interplant competition as a biasing factor in evaluating pre-treatment effects in cucumber. Scientia Hortic. 24: 115-122.

SpITTERs, C. J. T., 1979. Competition and its consequences for selection in barly breeding. Agric. Res. Rep. (Versl. landbouwk. Onderz.) 893: 268 pp.

SpitTers, C. J. T., 1984. Effects of intergenotypic competition on selection. In: W. LANGE, A. C. ZEven \& N. G. Hogenboom (Eds.), Efficiency in plant breeding: Proc. 10th Congr. Europ. Ass. Res. Pl. Breed., EUCARPIA. Pudoc, Wageningen, pp. 13-27.

SPITTERs, C. J. T. \& TH. KRAmER, 1985. Differences between spring wheat cultivars in early growth. Euphytica 34: 000-000. 